\title{
Modelling Machining-induced Residual Stresses after Laser-assisted Turning of Steels
}

\author{
Mohamed N. A. Nasr ${ }^{1,2, a^{*}}$, Mohamed Balbaa ${ }^{1, b}$ and Hassan Elgamal ${ }^{1, c}$ \\ ${ }^{1}$ Dept. of Mechanical Engineering, Faculty of Engineering, Alexandria University, Alexandria, Egypt \\ ${ }^{2}$ Dept. of Materials Science \& Engineering, Egypt-Japan University of Science \& Technology, \\ Alexandria, Egypt

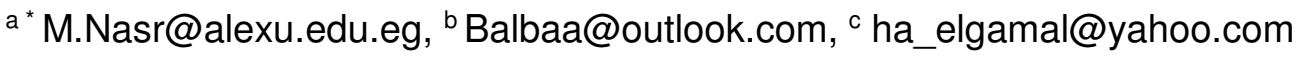

Keywords: Residual stresses, Laser-assisted machining (LAM), Finite element modelling (FEM), Turning, and Steels.

\begin{abstract}
The current study examines the effects of laser assistance on machining-induced residual stresses (RS), using finite element modelling, during turning of AISI 4340 steel at different feed rates. Dry orthogonal cutting was modelled, along with the pre-heating effect of the laser beam. Laser-assisted machining (LAM) resulted in higher surface tensile RS compared to conventional machining, with more pronounced effects at lower feed rates. This is basically because the assisted material experienced higher plastic deformation, due to thermal softening, as well as higher temperatures, which are both attributed to the pre-heating effect of LAM.
\end{abstract}

\section{Introduction}

Rapid developments in modern industries, especially for aerospace and nuclear applications, represent a challenge to the materials sector. Such applications typically require high strength alloys that can withstand severe operating conditions. This has been usually met with Ti- and/or Ni-based alloys, and in some cases using hardened steels. These alloys offer high strength, high hardness and resistance to high temperature and corrosion, but on the downside are hard to machine. Hard-tomachine materials typically have low thermal conductivity and diffusivity, which cause steep temperature gradients, and high tendency to strain hardening during machining. In addition, the presence of abrasive carbides and/or intermetallic phases, as well as adhesion to tool material adds to the complexity of the problem. This typically leads to excessive tool wear, premature tool cracking, built-up edge formation, high cutting forces, which are all reflected in lower metal removal rates and high cost [1 - 5].

Different methodologies have been developed to assist in overcoming machining issues of hard-to-machine materials. Two techniques were proved to be successful; cryogenic machining and laser-assisted machining (LAM). In cryogenic machining, liquid nitrogen in typically used (as a cooling-lubrication fluid) to significantly lower cutting temperatures and improve productivity and tool life [6]. On the other hand, LAM depends on pre-heating the workpiece material, using a laser beam, ahead of the tool. This results in a significant drop in cutting forces and tool wear due to thermal softening effects $[1-4,7]$.

Even though machining-induced residual stresses (RS) are very crucial in controlling part performance, the available literature paid very little attention to the effect of LAM on them. The literature mainly focused on tool wear, surface roughness, surface hardness, cutting forces, and chip formation. In an experimental study, Attia et al. [1] examined the effects of LAM on finish turning of Inconel-718, and found a significant drop in cutting forces compared to conventional machining (CM). Also, material removal rate increased by almost $800 \%$ and surface finish improved by about 25\%. Navas et al. [8] focused on age-hardened and solution-annealed Inconel-718, where the machinability of both alloys was found to improve, specially the former. Cutting forces dropped with higher laser power densities, higher cutting speeds, and when the distance between the laser spot and cutting tool decreased; however, a minimum distance was identified in order to avoid tool degradation by overheating. Anderson et al. [9] focused on tool wear, where they reported lower LAM flank wear compared to CM. Also, LAM specific cutting energy was significantly lower, and 
surface roughness was improved. In a study by Dumitrescu et al. [3], high power diode laser was used in turning hardened AISI D2 tool steel. LAM resulted in not only avoiding catastrophic tool failure, reducing thrust forces, but also increased tool life by about $100 \%$. Similar effects were found when machining different Ti-based alloys, as reported in [4].

Using finite element modelling (FEM) for examining the effects of LAM on machining parameters is actually very limited in the available literature. As an example, Bouchnak et al. [10] modelled LAM of $42 \mathrm{CrMo} 4$, where cutting forces dropped at different cutting speeds. In another study, Shi et al. [11] developed a FE model that included a new constitutive material model, friction model and heat transfer model. The authors also reported lower cutting forces for LAM; however, strain and strain rate fields were almost unaffected.

Ding and Shin [5] performed an experimental investigation on LAM when turning AISI 4130 steel. LAM resulted in lower cutting forces, higher surface tensile RS, but no effect was found on surface hardness; however, no explanation was provided. The authors also developed a threedimensional transient thermal model to predict temperature distribution. The model resulted in an "average material removal temperature" $\left(T_{m r}\right)$ that accounts for laser pre-heating effects, and depends on: 1) laser power, 2) cutting speed and 3) feed rate.

Based on the above, and to the authors' best of knowledge, very minimal focus was given to studying the effects of LAM on RS. Accordingly, the current work investigates the effects of LAM on RS, with special focus on surface RS, when turning AISI 4340 steel. A set of two-dimensional FE models was built in order to compare RS after LAM and CM, using the commercial FE software ABAQUS. Cutting conditions were chosen similar to those of Ding and Shin [5] for model validation.

\section{Finite Element (FE) Modelling}

Two sets of plane strain Lagrangian FE models were built, using the commercial FE software ABAQUS, in order to simulate the effects of laser assistance on RS when orthogonal dry cutting of AISI 4340 steel, under different feed rates. One set modelled CM and the other set modelled LAM, as explained below. Fig. 1 shows the cutting model geometry, along with applied boundary conditions. Each analysis consisted of two simulation steps; a cutting step followed by a relaxation step. The cutting step was solved using the explicit solver "ABAQUS/Explicit", while RS prediction was performed using the implicit solver "ABAQUS/Standard". This is because explicit solvers are more suitable for modelling the highly nonlinear cutting process; however, on the other hand, they extremely slow down the relaxation process [12]. In order to speed up the relaxation process even more, the time-efficient approach that was developed by one of the current authors [12] was used. Coupled temperature-displacement analysis was used in both steps, in order to allow for temperature-dependent properties and heat transfer. The current work was validated by comparing the predicted RS to experimental results, available in the literature, obtained under similar cutting conditions.

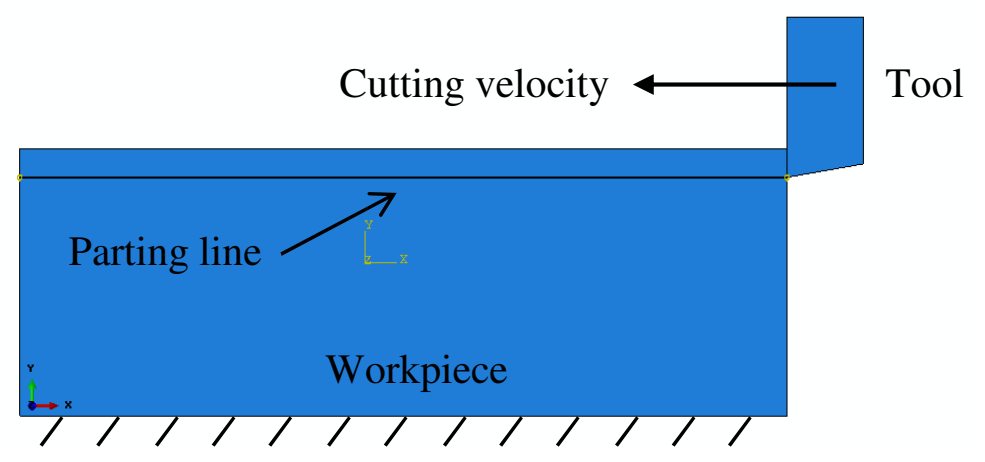

Fig. 1: Cutting model geometry and boundary conditions 
Workpiece Material Properties. AISI 4340 steel was selected for the current work, as an example for hard-to-machine materials. Physical properties were defined as function of temperature, and were obtained from [13]. The well-known Johnson-Cook $(\mathrm{J}-\mathrm{C})$ plasticity model, Eq. 1, was used to model the workpiece material plastic behaviour, where Table 1 shows the AISI 4340 values.

$$
\sigma=\left(A+B \varepsilon^{n}\right)\left(1+C \ln \left(\frac{\dot{\varepsilon}}{\dot{\varepsilon}_{0}}\right)\right)\left(1-\left(\frac{T-T_{r}}{T_{m}-T_{r}}\right)^{m}\right)
$$

Where:

$\sigma:$ Current von Mises flow stress

$A$ : Initial yield strength

$B$ : Strain hardening coefficient

$n$ : Strain hardening exponent

$m$ : Thermal softening exponent

$C$ : Strain rate coefficient $\varepsilon$ : Equivalent plastic strain

$\dot{\varepsilon}$ : Equivalent plastic strain rate

$\dot{\varepsilon_{0}}$ : Reference plastic strain rate

$T$ : Current temperature

$T_{r}$ : Reference temperature

$T_{m}$ : Melting temperature

Table 1: AISI $4340 \mathrm{~J}-\mathrm{C}$ constitutive model parameters [13]

\begin{tabular}{cccccc}
\hline$A[\mathrm{MPa}]$ & $B[\mathrm{MPa}]$ & $n$ & $C$ & $\dot{\varepsilon_{0}}\left[\mathrm{~s}^{-1}\right]$ & $m$ \\
\hline 950 & 725 & 0.375 & 0.015 & 1 & 0.625 \\
\hline
\end{tabular}

Chip Separation Criterion. For chip separation, material failure was predicted based on the cumulative damage law, given by Eq. 2. Damage occurs when the value of Eq. $2(D)$ reaches unity. The corresponding J-C damage parameters $\left(D_{1}-D_{5}\right)$ for AISI 4340 were obtained from [14], and presented in Table 2 .

Where:

$$
D=\Sigma\left(\frac{\Delta \bar{\varepsilon}}{\bar{\varepsilon}_{f}}\right)
$$

$\Delta \bar{\varepsilon}:$ Increment of equivalent plastic strain

$\bar{\varepsilon}_{\mathrm{f}}$ : Equivalent strain at failure

$$
\bar{\varepsilon}_{f}=\left(D 1+D 2 \exp \left(D 3 \frac{P}{\bar{\sigma}}\right)\right)\left(1+D 4 \ln \frac{\dot{\varepsilon}}{\dot{\varepsilon}_{0}}\right)\left(1-D 5\left(\frac{T-T_{r}}{T_{m}-T_{r}}\right)\right)
$$

$P / \bar{\sigma}:$ Ratio of hydrostatic pressure to equivalent stress

Table 2: AISI $4340 \mathrm{~J}-\mathrm{C}$ cumulative damage parameters (unit-less) [14]

\begin{tabular}{ccccc}
\hline$D_{1}$ & $D_{2}$ & $D_{3}$ & $D_{4}$ & $D_{5}$ \\
\hline 0.05 & 3.44 & -2.12 & 0.002 & 0.61 \\
\hline
\end{tabular}

Cutting Conditions. For model validation, the current cutting conditions were selected similar to those used by Ding and Shin [5], where experimental in-depth RS profiles (measured using X-ray diffraction) were presented for CM and LAM. A zero-rake PCBN insert and a cutting speed of 180 $\mathrm{m} / \mathrm{min}$ were used, along with two feed rates; $0.075 \mathrm{~mm} / \mathrm{rev}$. and $0.05 \mathrm{~mm} / \mathrm{rev}$. Tool physical properties were obtained from [15]. It is important to note that, the current work assumed sharpedged inserts in all simulations.

LAM Pre-heating Effects. In order to model the preheating effects of the laser beam, the LAM thermal model developed by Ding and Shin [5] was used in the current work. An initial temperature $\left(T_{L A M}\right)$ was assigned to the top layer of the workpiece, where cutting takes place. The value of $T_{L A M}$ was set equal to the average material removal temperature $\left(T_{m r}\right)$ defined in [5], explained above. For the current cutting conditions, the laser power was $1060 \mathrm{~W}$ and $865 \mathrm{~W}$ for $0.075 \mathrm{~mm} / \mathrm{rev}$ and 0.05 $\mathrm{mm} / \mathrm{rev}$ feed rates, respectively. This resulted in the same $T_{m r}\left(200{ }^{\circ} \mathrm{C}\right)$ for both feed rates. CM was simulated at room temperature $\left(T_{m r}=25^{\circ} \mathrm{C}\right)$. 
Friction and Heat Transfer. The simple Coulomb friction model was used to define friction between the tool and workpiece in the current work. Although it is a simple model, it has been widely used in metal cutting simulations [12, 13, 15 - 17]. This is because the real coefficient of friction cannot be measured any ways; besides, it gives reasonable results. A coefficient of friction of 0.15 was assumed in the current work. This is supported by having good agreement between the predicted and experimental RS profiles, as shown below. Based on material properties, $51.6 \%$ of the generated frictional heat was absorbed by the workpiece. For plastic deformation, $90 \%$ of its energy was assumed to be converted into heat. This value agrees well with the available literature $[12,13$, $15,18,19]$. Heat radiation and convection were neglected in the cutting model, as they are negligible compared to conduction. In the stress relaxation model, where the workpiece was left to cool down to room temperature, convection to air was considered with a coefficient of heat convection of $10 \mathrm{~W} / \mathrm{m}^{2}{ }^{\circ} \mathrm{C}$ and sink temperature of $25^{\circ} \mathrm{C}$.

\section{Results and Discussion}

The current section presents the effects of LAM on RS in cutting direction, as they typically dominate RS in other directions, especially in orthogonal cutting. Moreover, a special focus is given to the near-surface layer (with an average thickness of about $10 \mu \mathrm{m}$ ); i.e., surface RS rather than indepth RS, where tensile RS were found to be dominant. In addition, physical explanations are provided in terms of material plastic deformation and thermal softening ahead of the tool tip.

Model Validation. Fig. 2 and Fig. 3 compare the predicted and experimental RS profiles in cutting direction at feed rates $0.075 \mathrm{~mm} / \mathrm{rev}$ and $0.05 \mathrm{~mm} / \mathrm{rev}$, respectively. The experimental profiles were obtained from Ding and Shin [5], where similar cutting conditions were used and RS were measured using X-ray diffraction. It is worth mentioning that, the experimental work was actually performed on AISI 4130 steel, not AISI 4340. AISI 4340 steel was selected for the current work as it is a good example of widely used hard-to-machine steels, for which J-C model parameters are available in the literature. However, no experimental RS results are available for LAM of AISI 4340 in the literature. As mentioned earlier, the data available in the literature on the effect of LAM on RS is very limited. On the other hand, AISI 4130 steel is the only steel with currently available experimental RS results for LAM; however, its J-C model parameters are not available. Based on the similarity, demonstrated by Ding and Shin [5], between AISI 4340 and AISI 4130 in terms of X-ray parameters used for RS measurements, the available AISI 4130 experimental RS results [5] were used to validate the current AISI 4340 FE RS results.

In general, the predicted profiles followed the same trend as the experimental ones, starting with surface tensile RS, in all cases, and flipping to compressive RS within the top $10 \mu \mathrm{m}$. Focusing on the near-surface layer (top $10 \mu \mathrm{m}$ ), very good match between the predicted and experimental profiles is evident for the $0.075 \mathrm{~mm} / \mathrm{rev}$ case; however, FEM over predicted RS with a maximum difference of about 70-80 MPa for the $0.05 \mathrm{~mm} / \mathrm{rev}$ case. Such difference fits within the reported experimental error in measuring RS, which reached up to $\pm 100 \mathrm{MPa}$ in some cases [5]. It could be also partially attributed to the simplified friction model and / or the $T_{m r}$ model used in the current work. Far from the surface, deviation from experimental results is more evident. Further work is being currently conducted to improve in-depth prediction capabilities.

Effect of LAM on RS. As shown in Fig. 2 and Fig. 3, LAM resulted in higher surface tensile RS in cutting direction compared to CM. This is more evident at lower feed rates $(0.05 \mathrm{~mm} / \mathrm{rev})$. Such increase is mainly attributed to two reasons; 1 ) higher compressive plastic strains ahead of the tool tip, which tend to induce higher surface tensile RS; and 2) higher temperatures induced in the newly machined workpiece, which also have the same tendency for tensile RS. Fig. 4 and Fig. 5 present the predicted distribution of total plastic strain (which was mainly compressive) and temperature in the newly machined workpiece, respectively. Higher temperatures are a direct result to the preheating effect of LAM. At the same time, thermal softening results in easier and earlier plastic deformation ahead of the tool tip. However, no significant effect was noticed in the thickness of 
plastically deformed layer. The more pronounced effect of LAM at lower feed rates $(0.05 \mathrm{~mm} / \mathrm{rev})$ could be explained in terms of having a thicker pre-heated region ahead of the tool tip, as more time is available to the absorbed heat to propagate.

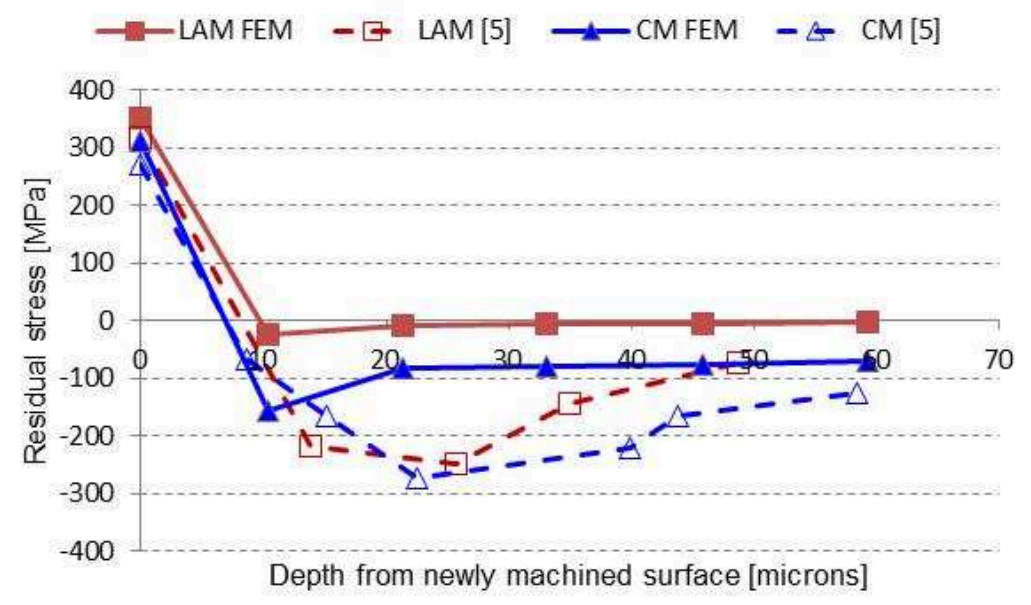

Fig. 2: Predicted and experimental RS profiles at $0.075 \mathrm{~mm} / \mathrm{rev}$.

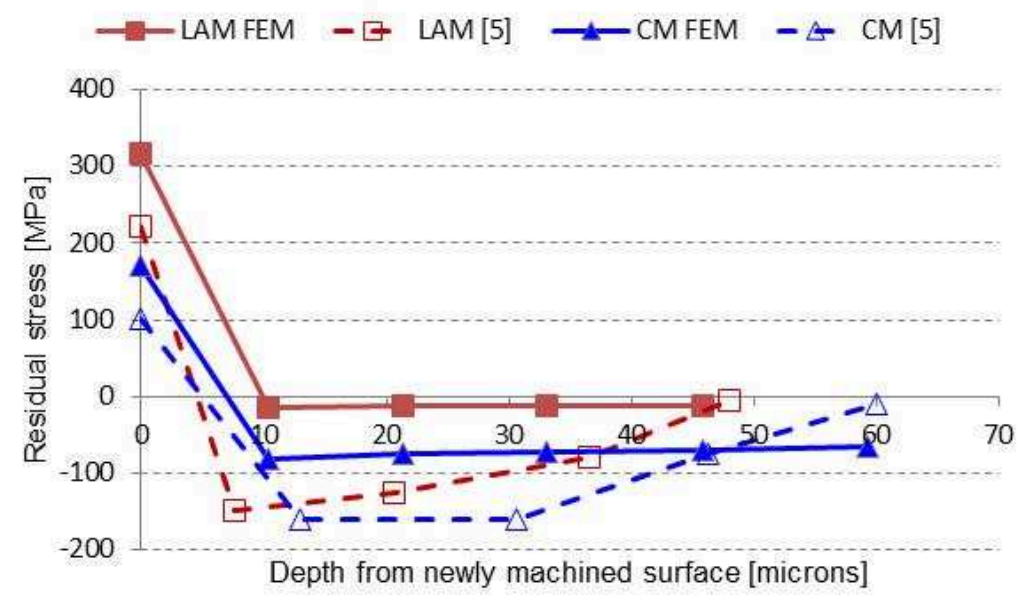

Fig. 3: Predicted and experimental RS profiles at $0.05 \mathrm{~mm} / \mathrm{rev}$.

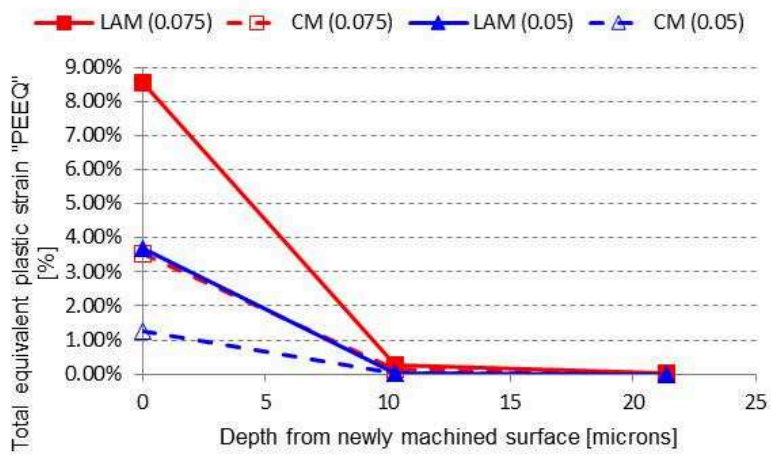

Fig. 4: Total equivalent plastic strain (PEEQ) distribution in machined workpiece

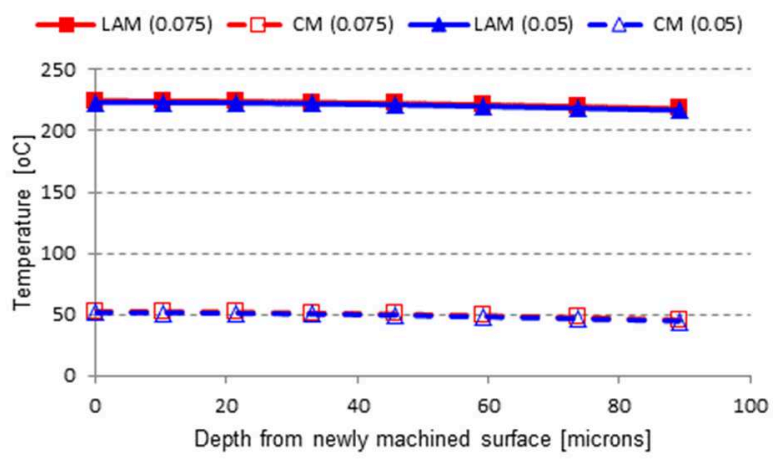

Fig. 5: Temperature distribution in machined workpiece after cutting

\section{Summary}

Dry orthogonal cutting of AISI 4340 was modelled using the commercial FE software ABAQUS, in order to examine the effects of LAM on RS and at different feed rates. The model was validated using experimental RS measurements available in the literature. For the examined cutting conditions, LAM resulted in higher surface tensile RS in the cutting direction compared to CM, with more pronounced effect at lower feed rates. The current results are attributed to the pre-heating effect of LAM, which resulted in higher temperatures and higher compressive plastic deformation ahead of the tool tip; both leading to surface tensile RS. 


\section{References}

[1] H. Attia, S. Tavakoli, R. Vargas and V. Thomson, Laser-assisted high-speed finish turning of superalloy Inconel718 under dry conditions, CIRP Ann. Manuf. Technol. 59 (2010) 83-88.

[2] S. Rajagopal, D.J. Plankenhorn and V.L. Hill, Machining aerospace alloys with the aid of 15 kW laser, J. of App. Metalworking, 2-3 (1982) 170-184.

[3] P. Dumitrescu, P. Koshy, J. Stenekes and M.A. Elbestawi, High-power diode laser assisted hard turning of AISI D2 tool steel, Int. J. Mach. Tool Manu. 46 (2006) 2009-2016.

[4] S. Sun, M.Brandt and M.S.Dargusch, Thermally enhanced machining of hard-to-machine materials - A review, Int. J. Mach. Tool Manu. 50 (2010) 663-680.

[5] H. Ding and Y. C. Shin, Laser-assisted machining of hardened steel parts with surface integrity analysis, Int. J. Mach. Tool Manu. 50 (2010) 106-114.

[6] J. Kenda, F. Pusavec and J. Kopac, Analysis of residual stresses in sustainable cryogenic machining of nickel based alloy - Inconel 718, J. Eng. Mater. Tech. 133 (2011) 1-7.

[7] G. Germain, P. DalSanto and J.L. Lebrun, Comprehension of chip formation in laser assisted machining, Int. J. Mach. Tool Manu. 51 (2011) 230-238.

[8] V. Navas, I. Arriola, O. Gonzalo and J. Leunda, Mechanisms involved in the improvement of Inconel 718 machinability by laser assisted machining, Int. J. Mach. Tool Manu. 74 (2013) 1928.

[9] M. Anderson, R. Patwa and Y.C. Shin, Laser-assisted machining of Inconel 718 with an economic analysis, Int. J. Mach. Tool Manu. 46 (2006) 1879-1891.

[10] T. B. Bouchnak, G. Germain, A. Morel and J.L. Lebrun, Influence of laser assistance on the machinability of the titanium alloy Ti555-3, Int. J. Adv. Manuf. Technol. 68 (2013) 24712481.

[11] B. Shi, H. Attia, R. Vargas and S. Tavakoli, Numerical and experimental investigation of laserassisted machining of Inconel 718, Mach. Sci. Technol. 12 (2008) 498-513.

[12] M.N.A. Nasr, E-G. Ng and M.A. Elbestawi, A modified time-efficient FE approach for predicting machining-induced residual stresses, Finite Elem. Anal. Des. 44 (2008) 149-161.

[13] M.N.A. Nasr, E-G. Ng and M.A. Elbestawi, Effects of strain hardening \& initial yield strength on machining-induced residual stresses, J. Eng. Mater. Tech. 129 (2007) 567-579.

[14] T. Mabrouki, J-F. Rigal, A contribution to a qualitative understanding of thermo-mechanical effects during chip formation in hard turning, J. Mater. Process. Technol. 176 (2006) 214-221.

[15] M.N.A. Nasr, E-G. Ng and M.A. Elbestawi, Effects of workpiece thermal properties on machining-induced residual stresses - thermal softening and conductivity, Proc. Inst. Mech. Eng., B J. Eng. Manuf. 221 (2007) 1387-1400.

[16] C.R. Liu and Y.B. Guo, Finite Element Analysis of the Effect of Sequential Cuts and ToolChip Friction on Residual Stresses in a Machined Layer", Int. J. Mech. Sc. 42 (2000) 10691086.

[17] H. Schmidt and J. Hattel, A Local Model for the Thermomechanical Conditions in Friction Stir Welding”, Modell. Simul. Mater. Sci. Eng. 13 - 1 (2005) 77-93.

[18] C. Shet and X. Deng, Residual Stresses and Strains in Orthogonal Metal Cutting, Int. J. Mach. Tool Manu. 43 (2003) 573-587.

[19] T.I El-Wardany, H.A. Kishawy and M.A. Elbestawi, Surface Integrity of Die Materials in High Speed Hard Machining. Part 2: Micro-hardness Variations and Residual Stresses, J. Eng. Mater. Tech. 122 - 4 (2000) 632-641. 